\title{
Première contribution à l'étude des Basidiomycètes de la région de Lalla Mimouna (Nord ouest du Maroc)
}

\author{
Ali OUTCOUMIT, Amina OUAZZANI TOUHAMI et Allal DOUIRA \\ Laboratoire de Botanique, de Biotechnologie et de Protection des Plantes, Faculté des Sciences, Université \\ IbnTofaïl, BP. 133, Kénitra, Morocco. \\ Auteur Correspondant : outcoumit1@yahoo.fr; douiraallal@hotmail.com
}

Original submitted in on 29th April 2013 Published online at www.m.elewa.org on 30 June 2013.

https://dx.doi.org/10.4314/jab.v66i0.94999

\section{RÉSUMÉ}

Objectif : Le travail présenté ici a pour objectif d'étudier la flore fongique de la région de Lalla Mimouna (Nord du Maroc).

Méthodologie et résultats: Des prospections effectuées dans la région de l'étude ont permis de noter des renseignements sur les espèces fongiques rencontrées. Au laboratoire, les critères macroscopiques et microscopiques d'identification de ces espèces ont été déterminés. Six espèces de Basidiomycètes Macromycètes ont été étudiées et décrites dans la région de Lalla Mimouna (Gharb-Nord) : Coltricia perennis (L.) Murrill, Phellinus torulosus (Pers.) Bourdot et Galzin, Pisolithus microcarpus (Cooke et Massee) G. Cunn., Tremella mesenterica Retz., Pluteus salicinus (Pers.) P. Kumm. et Clathrus ruber P. Micheli ex Pers. Ces deux dernières espèces peuvent être considérées comme espèces rares. La position systématique et l'écologie de toutes les espèces étudiées ont été discutées.

Conclusion et application de la recherche: Les résultats obtenus s'intègrent dans la contribution à la détermination de la diversité fongique du Maroc, qui demeure jusqu'à présent incomplète

Mots clés : Maroc, Lalla Mimouna, diversité fongique, Basidiomycètes.

First contribution to the study of basidiomycetes in the region of Lalla Mimouna (North West of Morocco)

Abstract

Objective: The present work is intended to study the fungal flora in the region of Lalla Mimouna (North of Morocco).

Methods and results: Surveys were done in the studied region allowed to note the details of the encountered fungal species. In the laboratory, the macroscopic and the microscopic criteria of these species were determined. Six species of Macromycetes Basidiomycetes were studied and described in the region of Lalla Mimouna (North of Gharb): Coltricia perennis (L.) Murrill, Phellinus torulosus (Pers.) Bourdot et Galzin, Pisolithus microcarpus (Cooke et Massee) G. Cunn., Tremella mesenterica Retz., Pluteus salicinus (Pers.) P. Kumm. and Clathrus ruber P. Micheli ex Pers. These two last species could be considered as a rare species. The systematic position and the ecology of all studied species have been discussed.

Conclusion and application of the research: The obtained results are integrated in the contribution to the determination of the fungal diversity in Morocco that remains incomplete until now. 
Keywords: Morocco, Lalla Mimouna, fungal diversity, Basidiomycetes.

\section{INTRODUCTION}

Dans le cadre de la contribution à la détermination de la diversité fongique du Maroc et à l'instar des autres régions favorables au développement des champignons supérieurs, des investigations ont été effectuées dans la région du Gharb-Nord (région de Lalla Mimouna) située dans le Maroc Atlantique Nord (Guérin, 1964). En effet, cette région se caractérise par un climat subhumide à composante océanique (Driouech, 2010). Le couvert végétal est constitué essentiellement de Quercus suber (forêt de Ferjana) et de plantations d'Eucalyptus (Benabid, 2000). Les études mycologiques de cette région ont connu une interruption depuis les travaux de Malençon et

\section{MATÉRIEL ET MÉTHODES}

Afin de contribuer à la détermination de la diversité fongique des Macromycètes de la région du GharbNord, des prospections ont été effectuées dans cette région du Nord - Ouest du Maroc. Ainsi, des investigations ont été réalisées sur différents substrats, parmi eux figurent Quercus suber et différentes espèces d'Eucalyptus. Sur le terrain, des renseignements relatifs à la saison de l'apparition des basidiocarpes et aux conditions climatiques qui caractérisent les périodes de cette apparition sont notés. Les caractères macroscopiques d'identification sont observés à l'œil nu et à l'aide d'une loupe à main et décrits. Ainsi, les dimensions des carpophores et de leurs parties anatomiques ont été mesurées. Les dimensions des basidiocarpes en $\mathrm{cm}$ sont déterminées selon leurs diamètres projetés sur un plan horizontal. Cette méthode consiste à exprimer les dimensions des basidiomes par le diamètre du cercle plus ou moins imparfait que dessine la partie la plus externe du pourtour du chapeau (Josserand, 1983). Egalement, l'aspect des basidiomes, la couleur du chapeau et de l'hyménophore, le type d'hyménophore; de même, l'épaisseur et la consistance de la chair, les ornementations des spécimens, leur odeur et leur saveur ont été déterminés. Les différents critères ont été étudiés sur des spécimens frais. Les renseignements recueillis sont notés in situ sur le carnet de récolte. Au laboratoire, les observations des différentes parties des carpophores ont été approfondies à l'aide d'une loupe binoculaire. La sporée a été obtenue par le dépôt des carpophores
Bertault (1970, 1975) et ceux de Bertault poursuivies jusqu'en 1972. Les prospections réalisées dans la région de Lalla Mimouna (Nordouest du Maroc) nous ont permis de rencontrer des Macromycètes Basidiomycètes et Ascomycètes. Dans cette première contribution, consacrée aux Basidiomycètes, six espèces appartenant aux groupes des Trémellomycètes, Agaricales, Hyménochaétales et aux Gastéromycètes ont été étudiées. La systématique de ces six espèces a été étudiée ainsi que leur importance pour les écosystèmes. L'écologie de chaque espèce a été discutée également dans ce travail.

mûrs sur des feuilles de papier de couleurs différentes et sur des lames de verre. La détermination de la coloration naturelle des spores a été réalisée par la comparaison de la coloration de la sporée avec la couleur de la craie blanche. Cette dernière est d'après Gilbert (1927), la couleur de référence pour les spores blanches. L'amyloïdité des spores est testée par l'addition d'une goutte d'eau iodée sur une pincée de la sporée. Des tests macrochimiques qui peuvent éventuellement donner des réactions colorées ont été effectués sur la chair. On a testé notamment l'action de l'eau iodée, $\mathrm{NaOH}(10 \%), \mathrm{FeSO}_{4}(10 \%)$ et $\mathrm{HNO}_{3}$ $(6 \%)$.Les critères microscopiques d'identification des espèces ont été déterminés à partir des coupes microscopiques au niveau des structures anatomiques. Ainsi, des coupes au niveau du revêtement, de la chair et de l'hyménophore ont été réalisées et observées sous microscope. L'observation des spores et la détermination de leur dimension ont été réalisées à partir des spores de la sporée. A l'aide du bout d'une lame de rasoir ou d'une aiguille lancéolée, nous prélevons par raclage une pincée de spores de la sporée. Celle-ci est déposée dans une goutte d'eau ou de liquide de montage. Les dimensions des spores ont été établies pour 20 spores de la sporée et données en micromètres $(\mu \mathrm{m})$. Les observations microscopiques ont été réalisées d'abord dans l'eau distillée puis dans le bleu coton, l'eau iodée, une solution aqueuse d'ammoniac (20\%) et dans le rouge Congo ammoniacal $(20 \%)$. Les dimensions des structures microscopiques ont été déterminées à l'aide d'un micromètre oculaire. 
Les grossissements utilisés sont 100,400 et 600 . La détermination de la forme des spores est obtenue à partir de l'utilisation du quotient sporique de Bas (1969). Des photographies ont été prises pour chaque espèce. Le biotope, les basidiomes, les critères macroscopiques et microscopiques du champignon ont été photographiés in situ et au laboratoire. L'étude systématique des espèces récoltées s'est basée sur l'habitat, l'écologie et sur les caractères macroscopiques des spécimens de chaque espèce. Les critères de détermination sont soigneusement notés. Au laboratoire, l'étude des échantillons est

\section{RÉSULTATS}

Tremella mesenterica Retz. 1769

Récolte du 16 février 2009, sur souches d'Eucalyptus sp. à Lalla Mimouna (Nord-Gharb) (Fig. 1). Les basidiomes $(1-8 \mathrm{~cm}$ de largeur $\times 0.5-3 \mathrm{~cm}$ de diamètre) se présentent sous forme de masses gélatineuses, cérébriformes, plissées, lobées, de couleur jaune pâle, jaune d'or ou jaune orangé. Les lobes ne sont pas bien individualisés et deviennent lisses avec l'âge. La chair est presque translucide et de consistance tendre et devient déliquescente. Au temps $\mathrm{sec}$, les fructifications se transforment en un mince film reviviscent au temps pluvieux. Aucune odeur ni saveur caractéristiques ne sont perceptibles. La sporée est blanchâtre à jaune pâle. L'hyménium est constitué de complétée par les données microscopiques et des réactions macro et microchimiques. La détermination des espèces rencontrées a été réalisée grâce aux clés de détermination (Heim, 1957, 1984; Kühner et Romagnési, 1984 ; Romagnési, 1995 ; de Courtecuisse et Duhem, 2000 et Poumarat, 2001).

La répartition des espèces au Maroc et dans le monde, les substrats sur lesquelles elles se développent, l'importance de chaque espèce pour les écosystèmes ainsi que leur importance gastronomique et pharmacomédicale ont été discutées. Dans ce travail, les espèces sont étudiées séparément.

basides à hypobasides globuleuses, subglobuleuses à ellipsoïdes. Les hypobasides portent quatre épibasides qui se prolongent par de longs stérigmates cylindriques. Les basides sont septées diagonalement ou en croix et bouclées à la base. Les spores (10 - $18 \mathrm{x}$ 8 - $12 \mu \mathrm{m})$ sont hyalines à jaunes, globuleuses, subglobuleuses ou ovoïdes à oblongues. Elles sont à paroi lisse et contiennent de grandes guttules. Les hyphes (1 $-3 \mu \mathrm{m}$ de diamètre) sont bouclés, à paroi mince et hyalines. Au stade jeune, avant la maturation des basides, une forme asexuée se développe sur le basidiome et forme des conidiophores qui libèrent des conidies $(2,5-3,5 \mu \mathrm{m})$ hyalines à jaunâtres dans l'eau. Les conidies sont subglobuleuses à ovoïdes. 

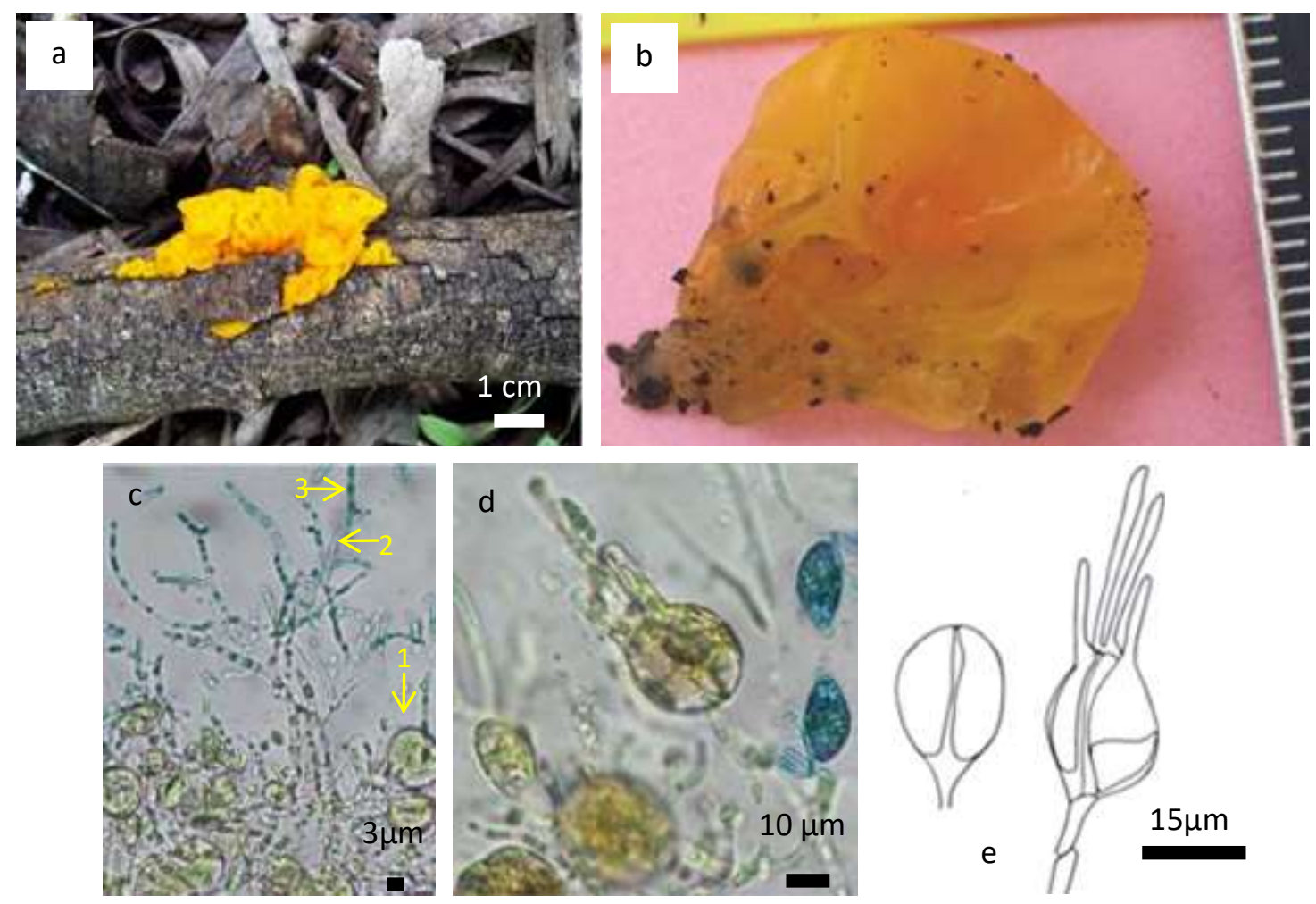

Figure 1 : Tremella mesenterica Retz - Un basidiocarpe cérébriforme jaune d'œuf sur un fissure d'une branche d'Eucalyptus (a) ; un basiodiocarpe dégagé de son substrat ligneux (b) ; jeunes basides (1), conidiophore (2) et conidies (3) (c) ; Basides à hypobasides et hypépibasides et basidiospores (d) et schéma d'une jeune baside et d'une baside mûre (e). Les observations microscopiques ont été réalisées dans l'eau au grossissement x 400 .

Pluteus salicinus (Pers.) P. Kumm. 1871

Récolte du 16 février 2009, sur souches d'Eucalyptus sp. à Lalla Mimouna (Gharb-Nord) (Fig. 2). Le chapeau $(3-6 \mathrm{~cm})$ est circulaire, parabolique à campanulé, puis devient convexe et enfin étalé. II est muni d'un mamelon central de $1 \mathrm{~cm}$ de diamètre environ et de couleur grise passant au gris foncé puis au gris sombre à noir. La surface du chapeau est sèche et de couleur grise, ou gris argentée, gris verdâtre à gris bleuâtre à l'état frais et légèrement hygrophane en gris clair à gris souris avec l'âge et à l'état sec. Elle est un peu squamuleuse au disque au niveau et autour du mamelon et très finement fibrilleuse, les fibrilles sont apprimées et de couleur sombre. La marge est circulaire, légèrement onduleuse, cannelée et non débordante. Le revêtement est adné et terne. L'hyménophore est constitué de lames libres, inégales, épaisses, subhorizontales ou subventrues, larges $(0,7$ $\mathrm{cm}$ ) et moyennement serrées. Les lames sont d'abord de couleur blanche, puis crème blanchâtre et à la fin deviennent de couleur saumon à rosâtre. L'arête est régulière à légèrement érodée et concolore aux faces des lames. La chair (6 $\mathrm{mm}$ au disque à moins d'un $\mathrm{mm}$ vers la périphérie) est de couleur blanche à nuance grisâtre dans le chapeau et blanche à nuance bleuâtre dans le pied. Le pied $(4-6 \times 0,3-0,6 \mathrm{~cm})$ est cylindrique, central, égal, plein et à base renflée en bulbe émarginé, blanc et rayé de fines fibrilles longitudinales, brunâtres qui sont mieux décelables à la loupe. Les fibrilles s'entrecroisent parfois entre elles en formant ainsi des formes polyhydriques.

La sporée est rose à rose brunâtre.

L'odeur et la saveur sont douces.

L'hyménium montre des basides (25 - 35 x 7 - $10 \mu \mathrm{m}$ ) cylindriques et tétrasporiques. Les cheilocystides (3560) $\times 21 \mu \mathrm{m}$ ) sont lagéniformes, piriformes ou parfois fusiformes et à contenu vésiculeux. Les pleurocystides $(95 \times 30 \mu \mathrm{m})$ sont abondantes, fusiforme-ventrues et surmontées d'un long col qui se termine au sommet par une base aplatie avec deux à cinq crochets. Les pleurocystides sont à paroi non épaisse et à contenu hyalin. Les spores $(8-10 \times 6,25-7,5)$ sont ovoïdes à elliptiques, non amyloïdes et à paroi lisse. 

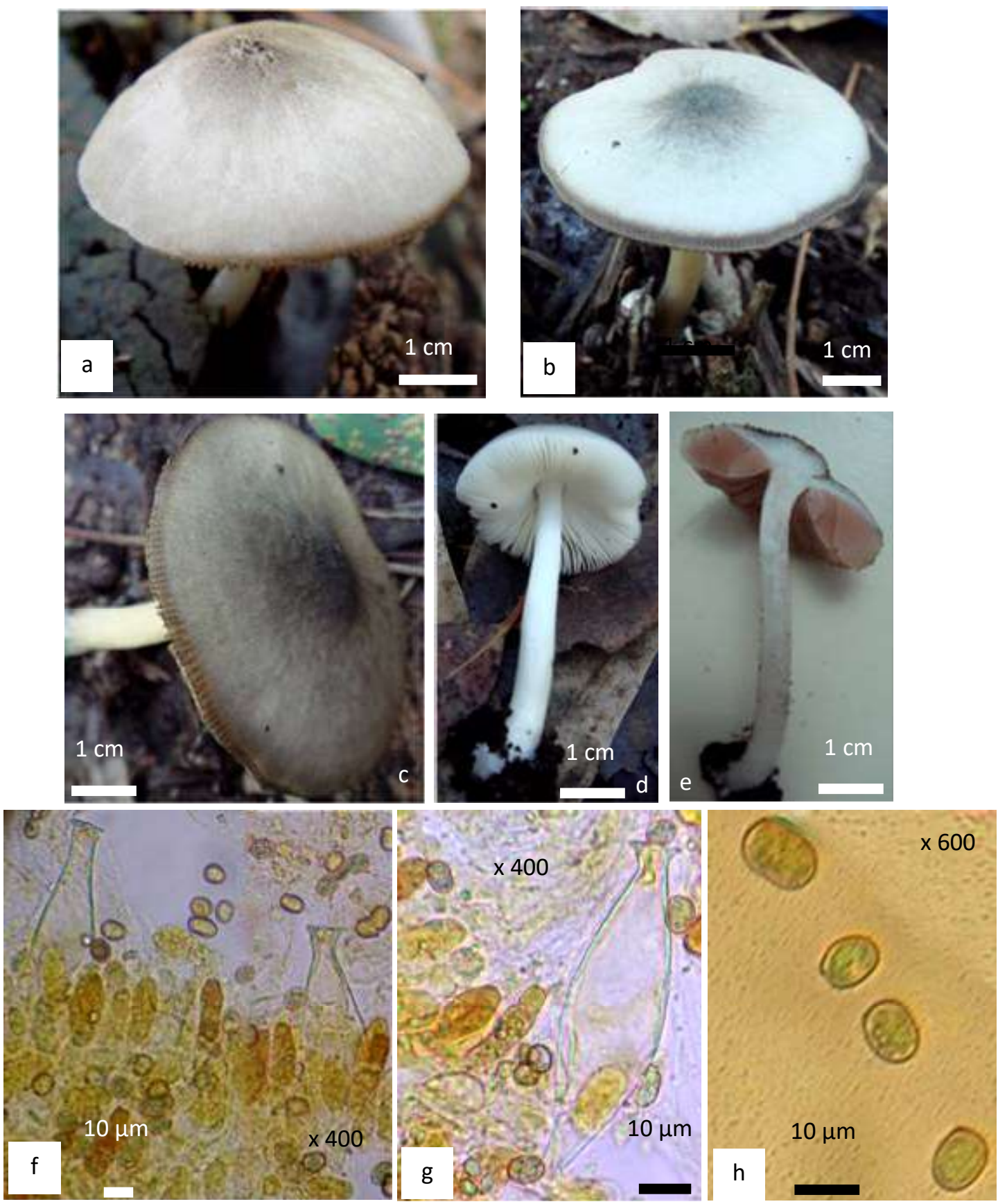

Figure 2: Pluteus salicinus (Pers) P. Kummel.- Basidiocarpe vouté à chapeau grisâtre et fibrilleux (a); bas idiome à chapeau gris verdâtre, mamelonné et plan (b); chapeau gris souris, mamelon noirâtre et marge cannelée (c); lames blanches, libres et pied blanc (d); chair blanche, lames roses et pied plein et marginé (e); hyménium à cystoïdes en crochets et basides cylindriques (f); une cystoïde en crochet à contenu hyalin ( $\mathrm{g}$ ) et basidiospores ovoïdes à elliptiques dans l'eau x 600 (h). ( $\mathrm{f}$ et $\mathrm{g}$ dans l'eau iodée).

Coltricia perennis (L.) Murrill 1903

Récolte du 9 janvier 2009, sur débris et feuilles de Quercus suber dans la forêt de Ferjana, Lalla Mimouna (Gharb-Nord) (Fig. 3.). Les basidiomes sont piliéés et stipités et souvent en amas soudés par la base des pieds ou / et par leurs chapeaux. Le chapeau (1 - 10 $\mathrm{cm}$ ) est plan ou déprimé en forme d'entonnoir et peut être ombiliqué. La surface du chapeau est mate, 
veloutée-tomenteuse sous la loupe à main, elle est constituée de bandes concentriques bien définies de couleur brun cannelle, beige, jaunâtre, brun jaunâtre, brun gris ou grise. La marge est mince, aigüe, incurvée, droite ou ondulée. La chair (1 à $3 \mathrm{~mm}$ ) est mince tenace et coriace chez les spécimens frais et elle est dure et cassante au desséchement ou avec l'âge. Elle est de couleur brune à brun rouille. L'hyménophore est tubulé-poré. Les pores ( 3 à $4 / \mathrm{mm}$ ) sont fins et de couleur brun pâle à brun cannelle ou chamois. Ils sont au début ronds, puis ils deviennent plus au moins allongés et enfin anguleux. Les tubes $(3 \mathrm{~mm}$ de hauteur) sont subdécurrents à décurrents et concolores aux pores. Le stipe $(0.5-5 \times 0,3-1 \mathrm{~cm})$ est central, cylindrique, plein, rigide, égal ou aplati et se termine par une base arrondie. II est tomenteux et de couleur brun orangé. La chair du pied est compacte et coriace. La sporée est de couleur brun jaunâtre.

La chair se colore en noirâtre sous l'action de $\mathrm{KOH}(3$ $\%)$.

L'odeur est nulle et la saveur est douce.

L'hyménium est constitué de basides (12 - $25 \times 5-7,5$ $\mu \mathrm{m})$ cylindracées ou claviformes à deux ou quatre basidiospores. Les spores $(6-8,5$ (9) x 4 - $5 \mu \mathrm{m})$ sont lisses et légèrement aplaties sur une face. Dans l'eau, les spores apparaissent hyalines à verdâtres au centre et orangées à la périphérie; dans l'eau iodée, les spores sont brunâtres (dextrinoïdes). Elles sont oblongues à elliptiques.
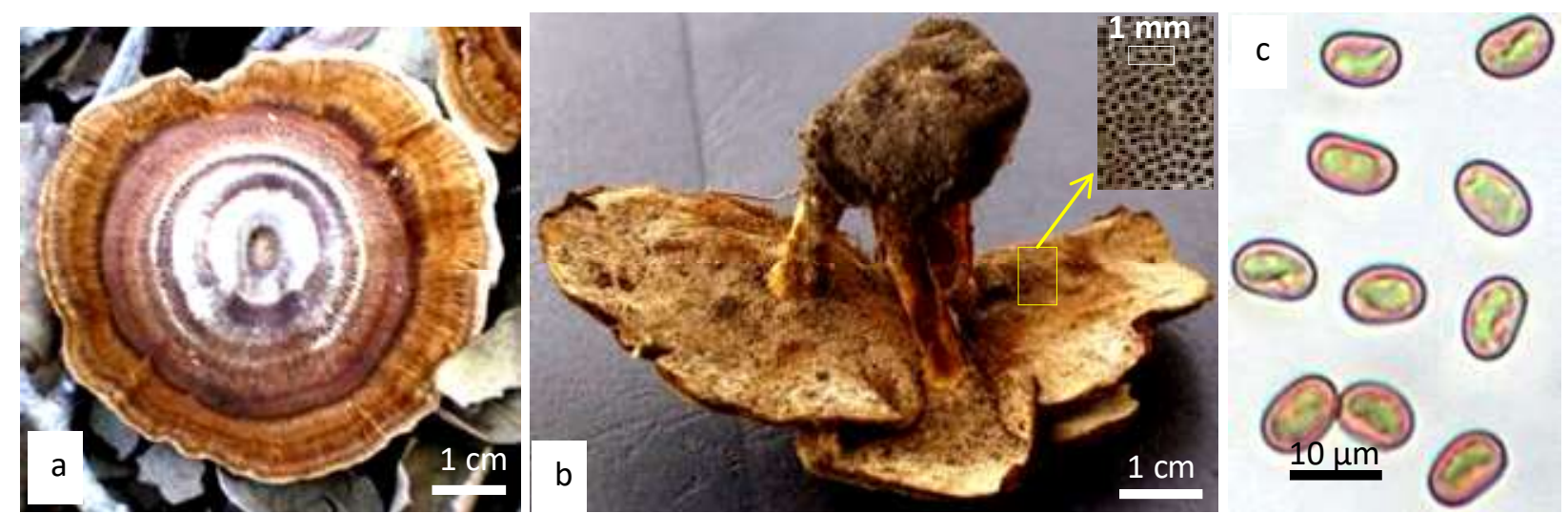

Figure 3 : Coltricia perennis (L.) Murrill : Un basidiome (in situ) à chapeau zoné concentriquement de couleur gris, chamois, brun, fauve, velouté - tomenteux, à marge claire et ondulée (a); touffes de spécimens collés par leurs chapeaux et leurs pieds et hyménophore tubulé - poré subdécurrent à décurrent et pores circulaires ou anguleux (b); basidiospores (c); les observations microscopique dans le bleu coton x 400 .

Phellinus torulosus (Pers.) Bourdot et Galzin 1925

Récolte du 15 février 2009: sur tronc d'Eucalyptussp.forêt de Ferjana, Lalla Mimouna (Gharb-Nord) (Fig.4.). Les basidiomes (10 - $35 \times 10$ $26 \times 1,2-5 \mathrm{~cm}$ ) sont vivaces, ligneux, pilée-sessiles ou collés au substrat ligneux par toute leur largeur. Ils sont plats à convexes, simples ou imbriqués. Le chapeau est de couleur brun jaunâtre, brun foncé à presque noire chez les vieux spécimens. II est zoné concentriquement, glabre à tomenteux surtout vers la base. Les bords sont entiers ou ondulés et de couleur brun jaunâtre. L' hyménophore est tubulé - poré. Les pores $(6-7 / \mathrm{mm})$ sont ronds et de couleur brun clair à jaune-brunâtre vers la marge. Les tubes (jusqu'à $5 \mathrm{~cm}$ ) sont de couleur brun jaunâtre pâle avec des tons blanchâtres inégalement répartis. La chair (jusqu'à 5 - 7 cm d'épaisseur) est de couleur brune, dure, ligneuse et légèrement zonée, avec une ligne noire qui la sépare de la surface du chapeau. Les hyphes génératifs (2,5 à $4 \mu \mathrm{m}$ de diamètre) sont cloisonnés, simples ou ramifiés et à paroi moyennement épaisse. Ils sont hyalins, jaunâtres ou brun jaunâtres et non amyloïdes. Les hyphes squelettiques ( $3-5 \mu \mathrm{m}$ de diamètre) sont non cloisonnés et à paroi épaisse $(1-1,5 \mu \mathrm{m})$. Ils sont de couleur brun rougeâtre et non amyloïdes.

Les cystides $(40-50 \times 10 \mu \mathrm{m})$ sont ventrues fusoïdes et entourées d'une paroi de 2 à $3 \mu \mathrm{m}$ d'épaisseur. Les basides $(20-30 \times 8-10 \mu \mathrm{m})$ sont droites, cylindracées et tétrasporiques.

Les basidiospores (4- $6 \times 3-4 \mu \mathrm{m})$ sont ovoïdes à elliptiques, hyalines et à paroi lisse. 

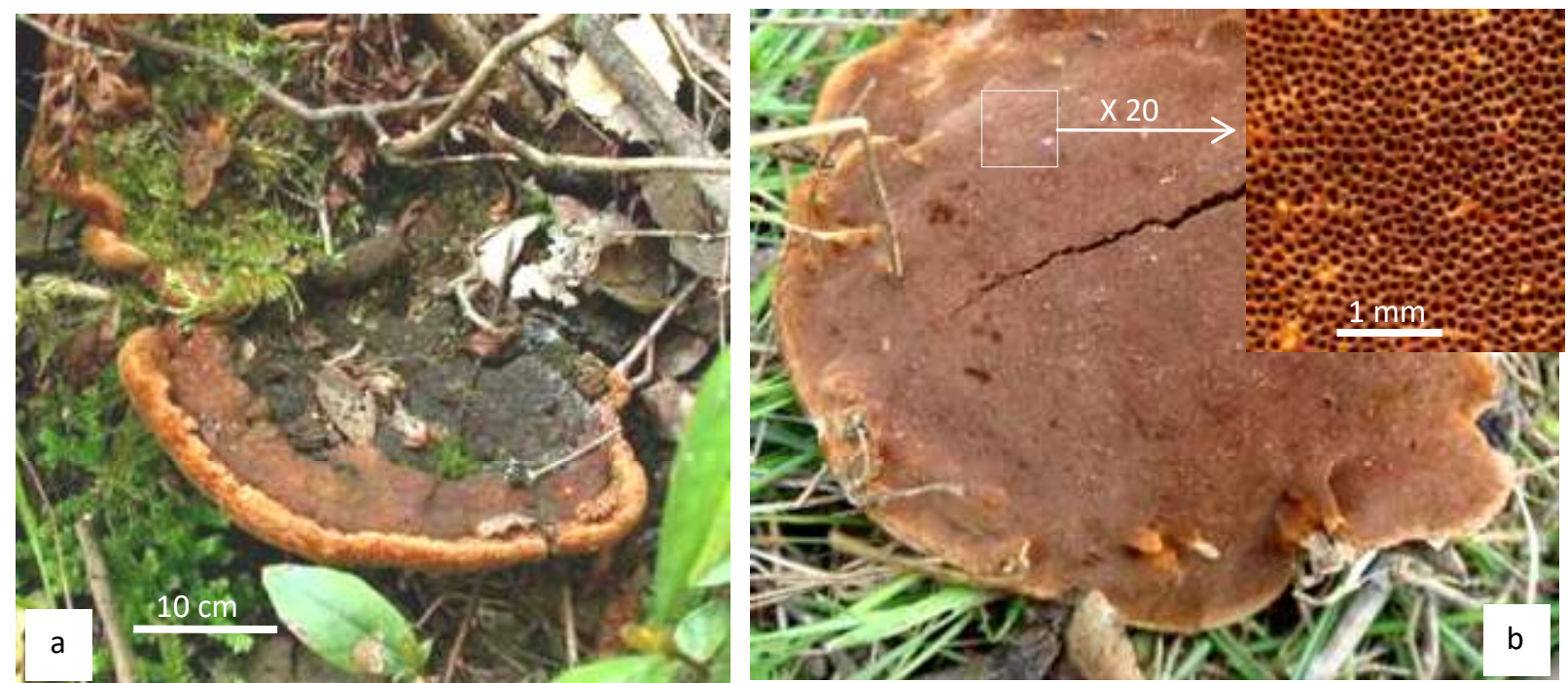

Figure. 4 :- Phellinus torulosus (Pers.) Bourdot et Galzin : Basidiome sur le col de Erica arborea (a) ; face hyméniale et pores agrandies 20 fois (b).

Pisolithus microcarpus (Cooke et Massee) G. Cunn. 1931

Récolté du 15 février 2009 sous Eucalyptus sp.: Région de Lalla Mimouna (Gharb-Nord) (Fig. 5). Les basidiomes de 5 à $12 \mathrm{~cm}$ en moyenne (parfois plus), sont semi-hypogés, de forme globuleuse, ovale ou irrégulière. Ils sont de couleur gris-brun, brun chocolat ou noirâtre. Le péridium est mince. La glèba est d'abord formée de logettes brillantes. A maturité, le péridium se déchire au sommet de façon irrégulière pour libérer les spores. La sporée est de couleur vert olive. L'intérieur des hyphes de la glèba, les basides et les ornementations des spores sont amyloïdes Les spores $(7-7,5 \times 7,5-7,75 \mu \mathrm{m})$ sont globuleuses à ovoïdes, verruqueuses et entourées de papilles. Elles sont brunes dans l'eau et leurs crêtes amyloïdes, se colorent en vert dans l'eau iodée. 

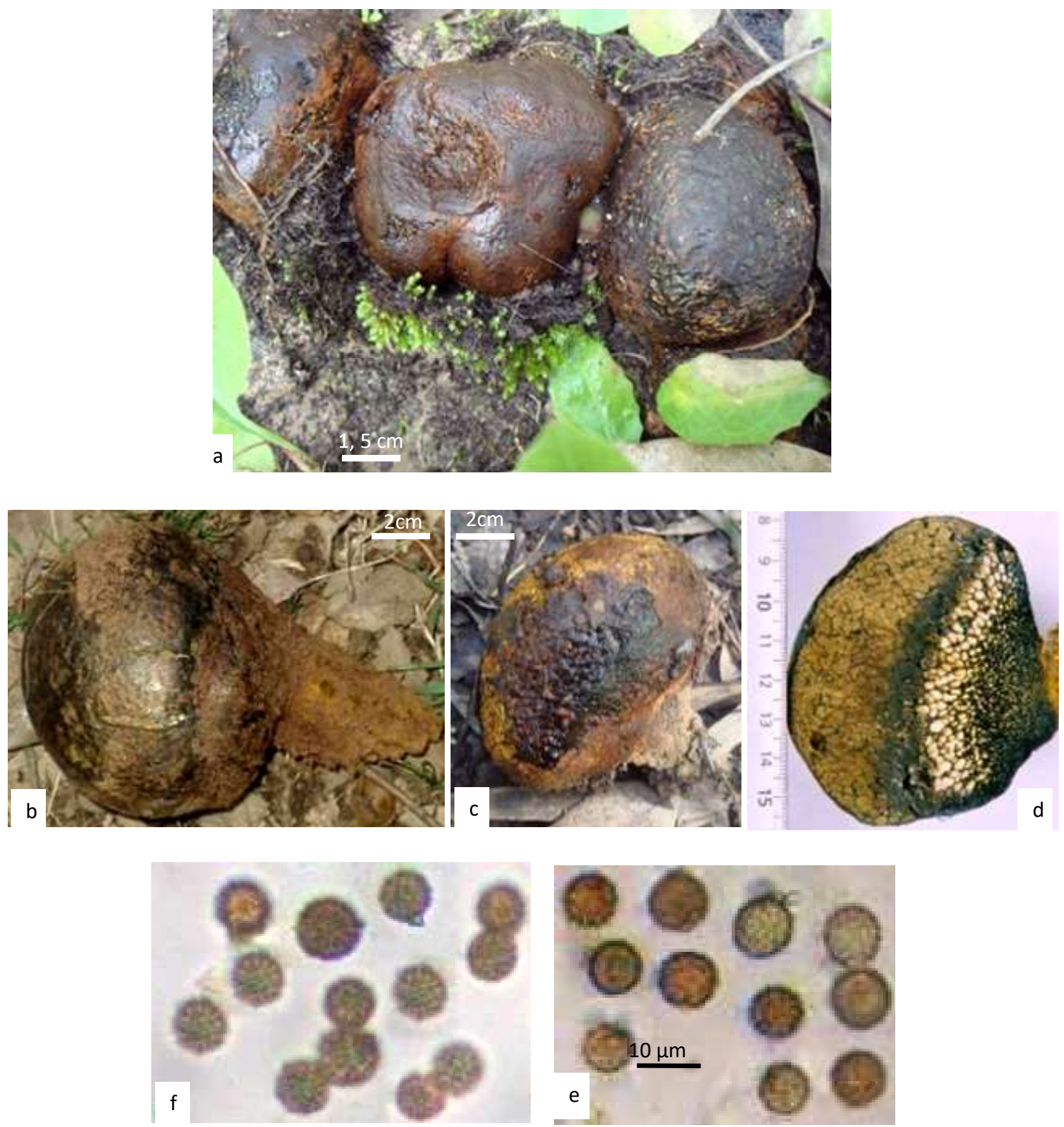

Figure 5 : - Pisolithus microcarpus (Cooke et Massee) G. Cunn. : Basidiomes à terre in situ, au pied d'un arbre d'Eucalyptus sp. (a); Deux basidiomes déterrés montrant la variation de la longueur du pseudostipe en fonction de la profondeur du substrat (b et c); coupe longitudinale d'un spécimen: La maturation des spores se fait du sommet vers la base (d); spores respectivement dans l'eau et dans l'eau iodée (x 400), les ornementations des spores sont amyloïdes. Les spécimens présentés ont été rencontrés près de Lalla Mimouna, le Gharb.

\section{Clathrus ruber P. Micheli ex Pers. (1801)}

Récolte du 9 janvier 2009, sous Eucalyptus sp.; Région de Lalla Mimouna (Gharb-Nord) (Fig. 6). Les deux spécimens de cette récolte sont au stade œuf et se trouvaient sous Eucalyptus sp. au bord de la route qui relie Moulay Boussalham et Lalla Mimouna (région de Lalla Mimouna), dans un endroit ombragé le 9 janvier 2009. Pendant cette période, la pluviométrie était importante et la température est moyenne pour la saison. Les fructifications sont sessiles, sous forme de 
sphères d'environ $7 \mathrm{~cm}$ de diamètre, blanchâtre, globuleuses à ovoïdes et grossièrement réticulées. Elles sont ancrées au sol par un gros cordon mycélien ramifié. L'exopéridium est blanchâtre à l'extérieur et de couleur crème à l'intérieur. En coupe, avant l'ouverture, la face interne montre des loges qui correspondent aux emplacements des bras du réceptacle. La chair est cassante, fragile, légère, transparente, caverneuse, gélatineuse et maculée sur la face interne. La déchirure du péridium laisse apparaitre le réceptacle sous forme de réseau sphérique constitué de mailles triangulaires à polygonales, allongées de couleur rouge corail puis orangées. Après l'ouverture de l'œuf, les mailles se dressent pour constituer un réseau sous forme grillagé (12 cm de hauteur environ). Au fur et à mesure que le champignon mûrit, les mailles pâlissent. La glèba est de couleur verdâtre à vert olive. Elle est sous forme de masse mucilagineuse, déliquescente. Elle est d'abord compacte, puis se dilate et prend la forme d'une mousse gluante à granulations sombres. L'odeur est forte, nauséabonde de charogne et attire les mouches. Les spores $(3.75-6.25 \times 1.5-2.5 \mu \mathrm{m})$ sont lisses, verdâtres, oblongues et elliptiques à boletoïdes.
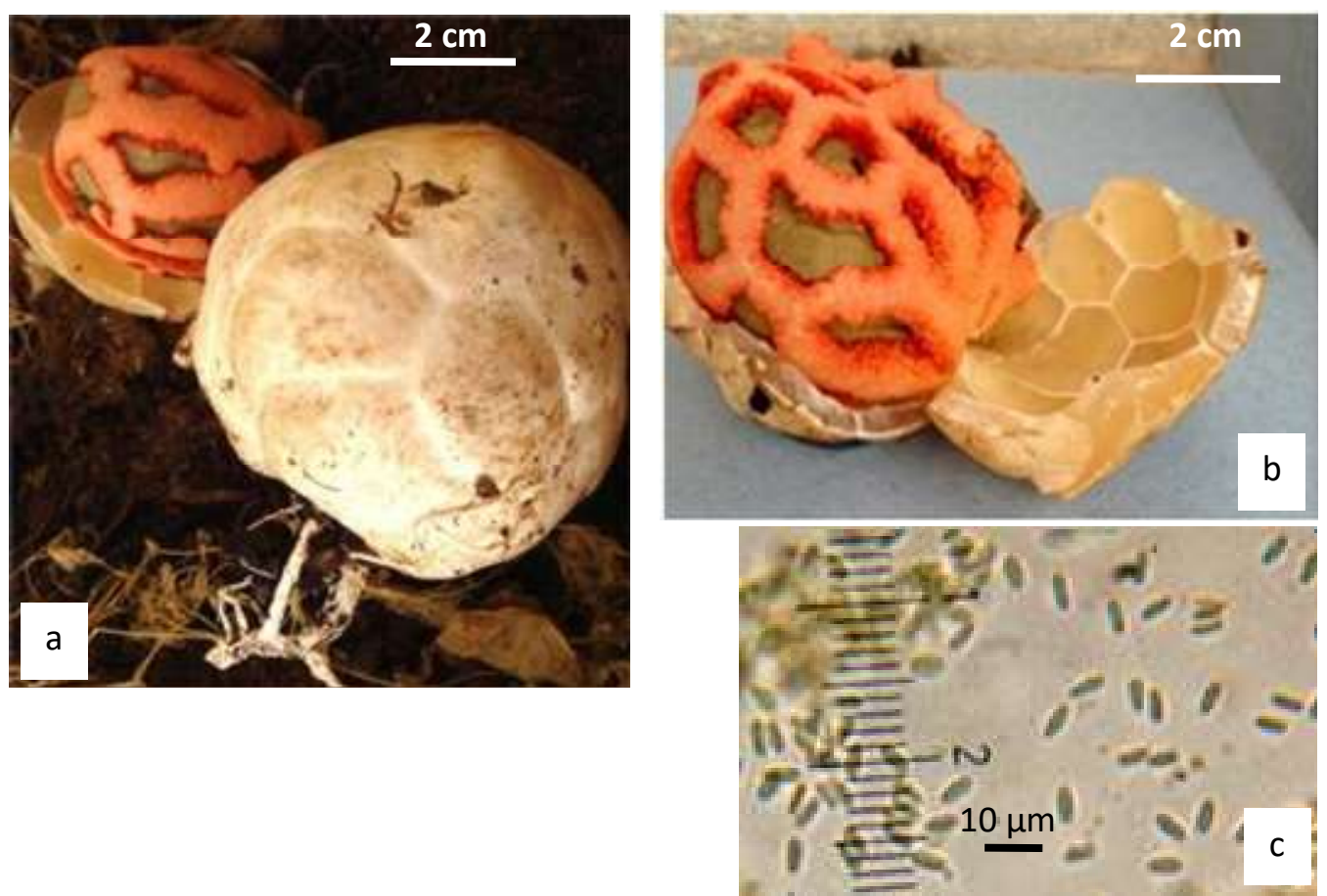

Figure 6 : Clathrus ruber P. Micheli ex Pers. (1801) : Stade œuf (a et b) et spores boletoïdes dans l'eau (c) (x 600).

\section{DISCUSSION}

Les Basidiomycètes de la région du Gharb ont été étudiés antérieurement, notamment par Maire et Werner (1937) et Malençon et Bertault (1970 et 1975). Cependant, la détermination de la diversité des champignons de cette région nécessite d'être complétée et mise à jour. Ainsi, les sorties mycologiques que nous avons effectué dans le GhardNord (région de Lalla Mimouna) nous ont permis de rencontrer des espèces nouvelles et des espèces non encore décrites pour cette région et également des espèces rares et menacées d'extinction. L'étude mycologique de ces espèces, la discussion de leur systématique sur les bases des critères anatomiques et phyllogénétiques et leur importance écologique, gastronomique et pharmacomédical ont été discutées. Ainsi :

Tremella mesenterica Retz. =Hormomyces aurantiacus Bonord. $1851=$ Tremella lutescens Pers. 1798 = Tremella mesenterica var. lutescens (Pers.) Pers. 1822 (Tremellaceae, Tremellales, Incertae sedis, Tremellomycètes, Agaricomycotina, Basidiomycota, Fungi) (Binder et al., 2005 ; Kirk et al., 2008) est une espèce qui appartenait aux Phragmo basidiomycètes à basides cloisonnées longitudinalement. Dans la nouvelle systématique, basée sur la phylogénie moléculaire, les Tremellales auquel appartient $T$. 
mesenterica ont été transférés dans la nouvelle famille des Tremellomycetes (Hibbett et al., 2007). Morphologiquement, T. mesenterica peut être confondue avec Dacrymyces palmatus (Schwein.) Bres., qui présente des basides en diapason (Gerhardt, 1999). T. mesenterica produit des conidies ovoïdes avant le développement des basides et la formation des spores (Hanet et Buttner, 2001). T. mesenterica se rencontre en solitaire sur le bois pourri, dans les fissures des branches tombées, des troncs et des souches des feuillus et mixtes des régions tempérées et tropicales en Afrique, en Asie, en Australie, en Europe et en Amérique du Nord et du Sud (Fenwick, 1995; Gerhardt, 1999). Au Maroc, T. mesenterica est également largement répandue. Elle a été signalée sur Quercus suber et sur Q. faginea, dans la forêt de la Mamora, le Moyen Atlas, le haut Atlas, la région de Zaiane et dans la région de Tanger (Maire et Werner, 1937; Malençon et Bertault, 1956). Nous avons rencontré T. Mesenterica dans la Mamora, à Khmis es-Sahel, dans la région de Lalla Mimouna et à Chefchaouenne (le Rif). T. mesenterica présente un potentiel lignocellulosique. En effet, elle dégrade la lignine du bois en décomposition, provoquant ainsi la pourriture blanche. Cette faculté est accompagnée de la production de l'éthanol à partir du bois, c'est une espèce doté du potentiel pour la production de la bioénergie (Houdou, 2004). Elle vit également en parasite du mycélium d'autres champignons, cas des espèces du genre Peniophora (Peniophoraceae, Aphyllophorales) (Pomperlau, 1980). T. mesenterica produit plusieurs molécules qui ont été brevetées comme des substances à intérêt médical. Ces molécules sont utilisées notamment pour la prévention $\mathrm{du}$ cancer, la stimulation du système immunitaire ( $y$ compris l'immunodéficience induite par le sida), la régulation de la glycémie et la régulation des taux de testostérone et d'œstrogènes (De Baets et al., 2002 et Lahayee, 2006). Bien que considérée comme sans saveur, T. mesenterica est comestible (Petersoon, 1818).

Pluteus salicinus (Pers.) P. Kumm. 1871 (Pluteaceae, Agaricales, Agaricomycetidae, Agaricomycetes, Agaricomycotina, Basidiomycota, Fungi) (Kirk et al., 2008) se rencontre dispersé ou solitaire sur les débris ligneux et sur le bois pourri de feuillus (Courtecuisse et Duhem, 2000). Au Maroc, P. salicinus a été uniquement signalé deux fois. Une fois sur une souche d'Eucalyptus à Tanger et l'autre sur Fraxinus angustifolia dans la région de Taza (Malençon et Bertault, 1970). Nous avons rencontré pour la première fois les six spécimens de cette espèce rare près de Lalla Mimouna (Gharb) en février 2009. P. salicinus est une espèce toxique et hallucinogène à cause de la présence dans ses basidiocarpes de la psilocybine et d'autres substances toxiques (Roux, 2006). C'est une espèce nouvelle pour le Gharb.

Coltricia perennis (L.) Murrill 1903 (Coltricia, Hymenochaetaceae, Hyménochaetales, Agaricomycètes, Basidiomycotina, Fungi) est connue sous différents synonymes: Boletus perennis 1753, Polystictus perennis (L.) Fr. 1879, Boletus subtomentosus Bolton 1788, Boletus cyathiformis Vill. 1789, Boletus leucoporus Holmsk 1790, Boletus fimbriatus Roth 1797, Boletus infundibulum Roth. 1797, Boletus confluens Schumach., Enum. pl. (Kjbenhavn) 1803, Coltricia connata Gray 1821, Polyporus perennis (L.) Fr. 1821, Polyporus perennis (L.) Fr. 1821 var. perennis, Boletus lejeunii L. Marchand, 1826, Boletus perfossus L. Marchand 1826, Trametes perennis (L.) Fr. 1849, Polyporus scutellatus I.G. Borshch. 1856, Pelloporus perennis (L.) Quél., 1886, Ochroporus perennis (L.) J. Schröt (1888) [1889], Xanthochrous perennis (L.) Pat., 1900, Microporus perennis (L.) Kuntze 1898, Polystictus prolifer Lloyd. 1908, Pelloporus parvulus Lázaro Ibiza 1916, Polystictus decurrens Lloyd., 1908, Polyporus parvulus Lázaro Ibiza 1916 (Kirk et al., 2008). Les basidiocarpes de ces espèces sont à stipes généralement central. Les hyphes sont monomitriques et les sois peuvent être absentes ou présentes. Leurs basidiospores sont dextrinoïdes chez certaines espèces et à maturité, elles deviennent jaune doré à brun rouille (Teixeira, 1962). C. perennis se rencontre en Europe, en Amérique et en Australie. Elle est souvent citée sous conifères, dans les brulis et dans les endroits perturbés. Au Maroc, $C$. perennis a été antérieurement rencontrée à Tanger par Malençon, 1956, cité par El -Assfouri (2003), dans le Rif (Malençon et Bertault, 1961, cités par Ait Aguil, 2005), dans la forêt de la Mamora (Malençon et Bertault, 1960, cités par El -Assfouri (2006) et dans la forêt de Ferjana à Lalla Mimouna (Bertault, 1972). Nous avons rencontré cette espèce en 2006 sous Quercus suber dans la forêt de la Mamora et en 2009 dans la forêt de Ferjana près de Lalla Mimouna.

Les espèces du genre Coltricia peuvent être rencontrées sur le sol ou sur le bois (Teixeira, 1962). Elle est également parfois considérée comme espèce ectomycorhizienne des pins (Baar et al., 1997, 1999).

Phellinus torulosus (Pers.) Bourdot et Galzin (= Fuscoporia torulosa (Pers.) T. Wagner et M. Fisch. 2001) est une espèce polyporoïde du genre Phellinus 
Quél. 1886 (Hymenochaetaceae, Hymenochaetales, Agaricomycètes, Agaricomycotina) (Hibbett et al., 2007 ; Kirk et al., 2008). C'est une espèce lignicole et thermophile qui se développe sur les racines, parfois à la base des troncs d'arbres et jusqu'à $2 \mathrm{~m}$ de hauteur (Tomšovsky et Jankovsky, 2007). Au Maroc, $P$. torulosus a été signalée sur plusieurs hôtes (Quercus rotundifolia, Q. suber, Eucalyptus globulus, Eucalyptus sp., Alnus glutinosa, Erica arborea, Acacia mollissima, Cupressus sp., Arbustus unedo et Chamaerops humilis) dans le Rif, la région de Tanger, la région de Larache, Lalla Mimouna, la forêt de la Mamora (Malençon, 1946, 1951, 1955, 1958 ; cités par ElAssfouri, 2003; Malençon et Bertault, 1960, cités par Ait Aguil, 2002; Bertault, 1964, 1965, 1967, 1972). Nous avons rencontré $P$. torulosus sur Eucalyptus globulus à Kénitra, sur Eucalyptus sp. dans la région de Lalla Mimouna et sur Erica arborea à Bab Taza dans le Rif. Quoique $P$. torulosus soit une espèce qui se développe sur une large gamme d'hôtes et que ses basidiomes peuvent se rencontrer sur les racines comme sur les troncs, l'analyse de l'ADN réalisé en 2007 par Tomšovsky et Jankovsky a montré qu'il s'agit de la même espèce. $P$. torulosus (Pers.) est phytopathogène, il cause la pourriture blanche des racines et des cols des arbres et des arbustes de nombreuses espèces (Campanile et Luisi, 2004). Toutes les espèces du genre Phellinus sont des parasites et / ou saprophytes sur le bois et provoquent de la pourriture blanche (Teixeira, 1962).

Pisolithus microcarpus (Cooke et Massee) G. Cunn. 1931. Les espèces du genre Pisolithus Alb. et Schwein. étaient classées dans la famille des Lycoperdaceae (Lycoperdales, Gasteromycètes (Clements et Shear 1954). Actuellement, la nouvelle systématique, basée sur les résultats de la biologie moléculaire, a transféré ce genre dans la famille Pisolithaceae puis à celle des Sclerodermataceae (Boletales, Agaricomycètes) (Binder et Bresinsky, 2002). Selon Kirk et al. (2008), dans la $10^{\mathrm{e}}$ édition du Dictionary of the Fungi, Pisolithus appartient aux Sclerodermataceae Corda (Boletales). Cette dernière famille se compose des genres Chloragaster Læssøe et Jalink 2004, Favillea Fr. 1848, Horakiella Castellano et Trappe 1992, Pisolithus Alb. \& Schwein. 1805 et Scleroderma Pers. 1801 (Cannon et Kirk, 2007). P. microcarpus se rencontre en abondance sous les Eucalyptus dans la forêt de la Mamora, dans la région de Sidi Yahia El- Gharb, près de Moulay Boussalham, à Lalla Mimouna et à Khmiss - es -Sahel (région de Larache). Les 23 espèces du genre Pisolithus sont associées aux racines des arbres par une symbiose mycorhiziènne. P. microcarpus est considéré comme espèce éctomycorhiziènne des Eucalyptus et des Pinus (Hitchcock, 2010).

Clathrus ruber Micheli ex Pers. (1801), connue sous plusieurs synonymes (C. cancellatus Tourn ex Fr., $C$. flavescens Pers., C. ruber var. flavescens (Pers.) Quadraccia et Lunghini, C. nicaeensis Barla et C. cancellatus Tourn. ex L. (1753)), est l'espèce type du genre Clathrus. L'espèce se rencontre également sous différentes formes et variétés, notamment, $\mathrm{C}$. ruber $\mathrm{f}$. kusanoi Kobayasi 1938, C. ruber f. ruber, P. Micheli ex Pers. 1801, C. ruber var. albus (Fr.) Quadr. \& Lunghini 1990, C. ruber var. ruber P. Micheli ex Pers. 1801 (Kirk et al., 2008). Auparavant, le genre Clathrus était classé dans la famille Clathraceae Chevall. 1826 (Dring, 1980 ; Poumarat, 2001). Récemment, Courtecuisse et Duhem (2000) l'ont classé dans la famille des Phallaceae Corda 1842. Les deux familles qui appartiennent à l'ordre des Phallales Fischer 1898 regroupent les espèces des Gastéromycètes dont les fructifications sont partiellement ou complètement souterraines et caractérisées par une glèba qui se dissout formant une masse visqueuse, généralement malodorante et rempli de spores (Bessey, 1952 ; Dring, 1980). Traditionnellement, la famille Clathraceae Ficher est étroitement liée à la famille Phallaceae Corda; les deux familles constituent ensemble l'ordre des Phallales (Pegler et Gomez, 1994). La distinction entre Phallaceae et Clathraceae est basée sur des critères morphologiques, principalement sur le support de la glèba. Dans la famille Phallaceae, la glèba est située à l'extrémité (non divisée) d'un long pseudostipe. La famille Clathraceae est caractérisée par une glèba qui est portée par des bras ou un grillage (bras anastomosés) surélevés ou non par un pseudostipe (Bessey, 1952; Demoulin, 1969 ; Dring, 1980 ; Pegler et Gomez, 1994; Poumarat, 2001). La taxonomie traditionnelle adoptée par les auteurs comme Zeller (1949) et Julich, (1981) a classé les espèces de la classe Gasteromycètes et leurs alliés les Gomphales dans des ordres différents plus ou moins éloignés comme les Lycoperdales, les Phallales et les Nidulariales. Les récentes recherches basées essentiellement sur les données de la biologie moléculaire indiquent que l'ordre des Phallales comprend également de nombreux champignons coralliformes, des espèces du genre Gomphus comme Gomphus floccosus et des Geasters comme Geastrum saccatum (Giachini, 2004 ; Hosaka et al., 2006). Pour ces auteurs, une nouvelle sous classe de Basidiomycètes est proposée; il s'agit des 
Phallomycetideae Hosaka, Castellano et Spatafora subclass. Nov. La sous classe des Phallomycetideae Hosaka, Castellano et Spatafora comprend les ordres Hysterangiales, Gomphales, Geastrales et Phallales. D'après Hosaka et al., (2006), ce groupe est l'équivalent des Phallales dans Dictionary of the Fungi et au clade Gomphoïde-Phalloïde sensu Hibbett et Thorn (2001). Pour Hibbett et Thorn (2001), les Homobasidiomycètes sont subdivisés en huit clades informels. L'ordre des Phallales est placé dans le clade Gomphoide-Phaloide. La sous classe des Phallomycetideae comprend quatre grands subclades (Gomphales, Hysterangiales, Geastrales et Phallales). De point de vue écologique, les taxons appartenant aux Phallomycetideae peuvent être ectomycorhiziens ou saprophytes. Selon la nouvelle classification (Hosaka et al., 2006), l'ordre des Phallales est composé des familles Phallaceae, Protophallaceae, Claustulaceae, Lysuraceae et Clathraceae. Le genre Clathrus est de nouveau reclassé dans cette dernière famille. Selon Dictionary of the Fungi (Kirk et al., 2001 et 2008), l'ordre des Phallales est classé dans la sous classe Basidiomycètes (Kirk et al., 2001) ou sous classe Phallomycetideae (Kirk et al., 2008), classe Agaricomycètes, subphyllum Agaricomycotina, phylum Basidiomycota dans le sous règne Dikarya et règne Fungi. L'aire de répartition de $C$. ruber s'étend de I'Afrique du Nord à l'Europe du Nord (Dring, 1980; Courtecuisse et Duhem, 1994) et se rencontre même en Amérique et en Australie (May et al., 2003). Bien que considérée principalement comme une espèce européenne, $C$. ruber a une distribution à plus grande échelle qui comprend I'Afrique du Nord, l'Asie et l'Australie. En dehors de la région méditerranéenne où l'espèce est moyennement fréquente (Roux, 2006), elle est considérée comme une espèce rare et sporadique ailleurs (Ivancevic et Tatic, 2004). Ainsi, dans la région de Styrie (Autriche), elle n'est mentionnée pour la première fois par Aron et Janoschek qu'en 2005. Parfois même, C. ruber est menacé d'extinction et figure dans la liste rouge comme en Bulgarie (Gyosheva et al., 2000) et en Ukraine (Hayova, 2010). En Amérique, C. ruber dont les gastérocarpes se rencontrent seuls ou grégaires à proximité des débris ligneux, dans les pelouses, les jardins et dans les sols cultivés, est supposée probablement introduite par l'activité humaine (Arora, 1979 - 1986). Au Maroc, C. ruber a été auparavant rencontré à Larache (Khmis es Sahel), à Tanger (Maire et Werner, 1937 ; Malençon et Bertault, 1957, 1968 et 1969 ; Bertault, 1972) et également au Plateau central (Malençon et Bertault,
1969). C'est une nouvelle espèce pour la région de Lalla Mimouna (Gharb).

C. ruber ne se rencontre au Maroc que, surtout, dans le Nord Ouest, sur le littoral atlantique et même dans ces régions, c'est une espèce peu commune voire même rare. En effet, comme nous pouvons le constater à partir de la consultation des anciens travaux (Maire et Werner, 1937; Malençon et Bertault 1957, 1968, 1969 ; Bertault, 1972 ; Ait Aiguil, 2002 et 2005 ; El Assfouri, 2006 ; Larouz, 2007; Haimed, 2007) et également à partir de nos investigations sur différentes régions du Maroc, C. ruber n'a été rencontré, depuis 1937 et jusqu'à maintenant que huit fois. C. ruber (P. Mich.) ex. Pers. n'a été rencontré au Maroc que rarement. Nous l'avons rencontré en 2009, pour la première fois dans la région de Lalla Mimouna. La fréquence de récolte de cette espèce est à notre estimation très basse comparativement aux fréquences observées pour d'autres Basidiomycètes. À noter à ce propos que $C$. ruber est un Gastéromycète et les espèces de cette division taxinomique sont normalement adaptées aux conditions relativement sèches. Ces conditions caractérisent beaucoup de régions du Maroc et théoriquement la fréquence des récoltes devrait être nettement supérieure. L'habitat de $C$. ruber, au moins dans le tangérois (Tanger à Larache) et même dans la région de Lalla Mimouna est fortement menacé par l'activité humaine (construction urbaine, route, etc.). Aucune précision concernant le lieu de la récolte de $C$. ruber dans le plateau central en 1969 n'a été fourni par les auteurs. Ainsi, aucune hypothèse ne peut être formulée quant au devenir du lieu de la dite récolte. Mais, l'espèce n'est signalée qu'une seule fois dans cette région et nous pensons que c'est déjà assez suffisant pour confirmer sa rareté également dans les subéraies de la Meseta.

D'après ces données bibliographiques et d'après nos investigations au Maroc, $C$. ruber peut être considérée comme une espèce rare, voir même menacée d'extinction; d'une part par le nombre restreint de ses stations et la fréquence faible des récoltes et d'autre part, les biotopes de l'espèce sont eux-mêmes, quand ils existent encore, sévèrement menacés. Ces deux critères sont tenus en compte pour l'inscription d'une espèce sur une liste rouge (Courtecuisse, 1997). Au Maroc, l'établissement d'une liste rouge n'est pas encore possible vu qu'un inventaire des Basidiomycètes n'est pas encore achevé et que la diversité de la mycoflore marocaine n'est pas encore bien déterminée jusqu'à maintenant. Mais, une attention particulière est nécessaire pour protéger les 
espèces vulnérables et menacées comme $C$. ruber. $C$. ruber est une espèce saprophyte, thermophile, préférant les lieux ombragés et humides. Elle se développe souvent dans les zones côtières sur des sols sablonneux et calcaires, dans les forêts et dans les parcs (Giachini, 2004). D'après Marchand (1975), l'œuf

\section{CONCLUSION ET PERSPECTIVES}

Six espèces de Basidiomycètes, rencontrées dans la région du Gharb-Nord, domaine du Maroc Atlantique Nord, ont été présentées dans cette étude. Tremella mesenterica (Trémellomycètes), Pluteus salicinus (Agaricales), Coltricia perennis, Phellinus torulosus (Hymenochaetales), Pisolithus microcarpus et Clathrus ruber (Gastéromycètes) ont été décrites pour la première fois dans la région de Lalla Mimouna.

Les espèces présentées dans ce travail sont caractérisées par des rôles importants et diversifiés. En effet, Tremella mesenterica présente un potentiel lignocellulosique. Elle dégrade la lignine du bois en décomposition, provoquant ainsi la pourriture blanche. Cette faculté est accompagnée de la production de l'éthanol à partir du bois, c'est une espèce dotée du potentiel pour la production de la bioénergie. Elle vit également comme parasite du mycélium d'autres champignons, comme les espèces du genre Peniophora (Peniophoraceae, Aphyllophorales). T. mesenterica produit plusieurs molécules à intérêt

\section{BIBLIOGRAPHIE CITÉE}

Ait Aguil F., 2005. - Contribution à l'étude de la biodiversité de la flore fongique des forêts marocaines: Cas des Basidiomycètes du Rif Oriental et du Rif Occidental et des Gastéromycètes de la Mamora. Thèse de Doctorat, Université Ibn Tofaïl, Faculté des Sciences, Kénitra, Maroc, $127 \mathrm{p}$.

Ait Aguil F., El Houssni A., Ouazzani Touhami A., Douira A., 2002 - Contribution à la connaissance des Gastéromycètes (Basidiomycètes) de la forêt de la Mamora. Ann. Rech. For. Maroc, Tome 30: 77-87.

Aron A. et Janoschek R., 2005 - Der Scharlachrote Gitterling Clathrus ruber (Micheli) Persoon. Erstfund in der Steiermark Joannea Bot., 4: 41-44.

Arora D., 1979 - 1986 - Mushrooms demystifield: a comprehensive guide to the fleshy fungi. Printed and bound in China, $927 \mathrm{p}$.

Baar J., Comini B., Oude Elferink M. et Kuyper Th. W., 1997 - Performance of four ectomycorrhizal de C. ruber, comme ceux de C. archeri ou Phallus impudicus peut être consommé, une fois débarrassé de sa partie gélatineuse. II présente en effet la saveur et l'odeur de radis. Mais après la déchirure de l'exopéridium, l'odeur fétide et répugnante de ces espèces les rend non comestibles.

médical. Bien que considérée comme sans saveur, $T$. mesenterica est comestible. Pluteus salicinus est une espèce saprophyte, toxique, hallucinogène. C'est une espèce rare et nouvelle pour le Gharb. Coltricia perennis peut être rencontrée sur le sol ou sur le bois. Phellinus torulosus est quant à elle une espèce phytopathogène qui cause la pourriture blanche. Pisolithus microcarpus est une espèce ectomycorhizienne de l'Eucalyptus et $C$. ruber est une espèce des sols sablonneux et calcaire. Pluteus salicinus et Clathrus ruber sont deux espèces rares et nouvelles pour le Gharb. Ainsi, les rencontrer contribue à mieux connaitre la diversité des Basidiomycètes de région du Gharb du Maroc. Ultérieurement, d'autres espèces Basidiomycètes et Ascomycètes rencontrées dans la région du Gharb-Nord et non décrites dans les travaux antérieurs seront présentées. Egalement, nous envisageons de compléter l'inventaire des Macromycètes de cette région.

fungi on organic and inorganic nitrogen sources. British Mycological Research, 101(5): 523-529.

Baar J., Horton T. R., Kretzer A. M. et Bruns T. D., 1999 - Mycorrhizal colonization of Pinus muricata from resistant propagules after a stand-replacing wildfire. New Phytologist, 143: 409-418.

Bas C., 1969 - Morphology and subdivision of Amanita and a monograph of its section Lepidella. Persoonia, 5 (4) : 285-588.

Benabid A., 2000 - Flore et écosystèmes du Maroc. Evaluation et préservation de la biodiversité. Éd. Ibis Presse, Paris, $359 \mathrm{p}$.

Bertault R., 1964 - Listes des champignons récoltés à Perdicaris. Comptes rendus mensuels des Séances de la Société des Sciences Naturelles et Physiques du Maroc, T 30, presses des éditions Marocaines et Internationales, Tanger, Maroc, pp 219 - 38. 
Bertault R., 1965 - Comptes rendues de la Société des Sciences Naturelles et Physiques du Maroc. Presses des éditions Marocaines et Internationales, Tanger, tome 31, pp $42-43$.

Bertault R., 1967 - Comptes rendues mensuels des séances de la Société des Sciences Naturelles et Physiques. Presses des éditions Marocaines et internationales, Tanger, T. 33, p 54.

Bertault R., 1968 - Centre de Tanger : Excursions au Daharzhiro, à la Cuesta Colorada, à Khmis-esSahel (2), à Larache Dar Chaoui et à Cherf-elAkab. T 34, Presses des éditions Marocaines et Internationales, Tanger, Maroc. pp 18-28.

Bertault R., 1972 - Centre de Tanger : Excursions à Cherf-el-Akab, Perdicaris, à la Cuesta Colorada, El-Araïch, Khmis-de-Sahel, Jbel Kbir, et forêt de Ferjana. Comptes redus mensuels des Séances de la Société des Sciences Naturelles et physiques du Maroc. T. 38, Presses des éditions Marocaines et Internationales, Tanger, Maroc, pp 121-145.

Bessey E. A., 1952 - Morphology and Taxonomy of Fungi. Constable and Compagny limited, London, W. C., 2, 791 p.

Binder M. et Bresinsky A., 2002 - Derivation of a polymorphic lineage of Gasteromycetes from boletoid ancestors. Mycologia, 94 (1): 85 - 98.

Binder M., Hibbett D. S., Larsson K. H., Larsson E., Langer E. et Langer G., 2005 - The Phylogenetic distribution of resupinate forms across the major clades of mushroom forming fungi (Homobasidiomycetes). Systematics and Biodiversity, 3 (2): 113-157.

Campanile G et Luisi N., 2004 - The occurrence of Phellinus torulosus in Apulia and Basilicata (Southern Italy): identification of isolates by morphologic, microscopic, and molecular means. Phytopathologia Mediterranea, 43 (2): 289-298.

Cannon P.F. et Kirk P.M., 2007 - Fungal Families of the World. CAB International Wallingford, pp 313 314.

Clements F. E. et Shear C. L., 1954 - The genera of fungi. Ed. Hafner publishing Co., New York, $496 \mathrm{p}$.

Courtecuisse R. 1997. - Liste rouge des champignons menacés de la région Nord-Pas-de-Calais (France). Cryptogamie Mycologie, 18 (3) :183219.
Courtecuisse R. et Duhem B. 1994 - Guide des champignons de France et d'Europe. Ed. Delachaux et Niestle, Lausanne, $260 \mathrm{p}$.

Courtecuisse R. et Duhem B. 2000 - Guide des champignons de France et d'Europe. Ed. Delachaux et Niéstlé, Lausanne, 476 p.

De Baets S., du Laing S., François C. et Vandamme E. J., 2002 - Optimization of exopolysaccharide production by Tremella mesenterica NRRL Y6158 through implementation of fed-batch fermentation. Journal of Industrial Microbiology and Biotechnology, 29 (4) : 181 - 184.

Demoulin V., 1969 - Introduction à l'étude des Gastéromycètes de Belgique. Ed. Naturalistes Belges, Bruxelles, $59 \mathrm{p}$.

Dring D.M., 1980 - Contributions towards a rational arrangement of the Clathraceae. Kew Bulletin, 35: $1-96$.

Driouech F., 2010 - Distribution des précipitations hivernales sur le Maroc dans le cadre d'un changement climatique : descente d'échelle et incertitudes. Thèse de Doctorat de l'Université de Toulouse, $164 \mathrm{p}$.

El - Assfouri A., 2006 - Biodiversité fongique du Maroc : Etude des Basidiomycètes de la forêt de la Mamora. Thèse de Doctorat, Université Ibn Tofaill, Faculté des Sciences, Kénitra, Maroc, $251 \mathrm{p}$.

El-Assfouri A., Ouazzani Touhami A., Fennane M., Zidane L. et Douira A., 2003 - Inventaire des spécimens fongiques de l'Herbier national de I'Institut Scientifique de Rabat. Bulletin de I'Institut Scientifique, Rabat, section Sciences de la Vie, $25: 1$ - 23.

Fenwick G. A., 1995 - Tremella mesenterica in laboratory culture. Mycologue 9 (3) : 124-126.

Gerhardt E., 1999 - Guide Vigot des champignons. Ed. Vigot, Paris, $714 \mathrm{p}$.

Gerhardt E., 1999 - Guide Vigot des Champignons. Ed. Vigot, Paris, $713 p$.

Giachini A. J., 2004 - Systematics, phylogeny, and ecology of Gomphus sensu lato. Dissertation for the degree of Doctor of Philosophy in Forest Science. Oregon State University, 446 p.

Gilbert E. J., 1927 - Les livres du mycologue. Tome I : la spore des champignons supérieurs, couleur, forme, ornementation, terminologie, valeur taxonomique. Eds. Librairie E. Le François, Paris, $219 \mathrm{p}$.

Guérin J.C., - 1964 - La mise en valeur et 
l'aménagement des subéraies marocaines. Revue forestière française, 1: $4-32$.

Gyosheva M., Fakirova V. \& Denchev C., 2000 - Red list and threat status of Bulgarian macromycetes. Historia Naturalis Bulgarica, 11: $139-145$

Haimed M., 2007 - Biodiversité fongique du Maroc: Etude des champignons Basidiomycètes du plateau central et des jardins exotiques. Thèse de Doctorat. Université Ibn Tofaill, Faculté des Sciences, Kénitra, Maroc, 273p.

Hanet P. et Buttner R., 2001 - Mansfelds of agricultural crops : (Except ornamentals) Santa Clara, CA : Springer- VerlagTellos, $p 23$.

Heim R., 1957 - Les champignons d'Europe. Tome II. Partie descriptive: Basidiomycètes. Ed. N. Boubée et Cie. Paris (VI), $570 \mathrm{p}$.

Heim R., 1984 - Champignon d'Europe, Généralités Ascomycètes- Basidiomycètes. Nouvelle des éditions Boubée, Paris, $680 \mathrm{p}$.

Hibbett D. S., et Thorn R.G., 2001 - Basidiomycota: Homobasidiomycètes In: Mc Laughlin D.J, Mc Laughlin E.J., Lemke P.A. (eds.). The Mycota vol. VII, Part B., Systematics and Evolution. Springer Verlag, Berlin, pp 121-168.

Hibbett D.S., Binder M., Bischoff J. F., Blackwell M. Cannon P. F., Eriksson O. E., Huhndorf S., Timothy J., Kirk P.M., Locking R.L., Thorsten Lumbsch H., Lutzoni F., Matheny P.B., McLaughlin D.J., Powell M.J., Redhead S., Schoch C.L., Spatafora J.W., Stalpers J.A., Vilgalys R., Aime C.R., Aptroot A., Bauer R., Begerow D., Benny G.L., Castlebury L.A., Crous P.W., Dai Y-C., Gams W., Geiser D.M., Griffith G.W., Gueidan C., Hawksworth D.L., Hestmark G., Hosaka K., Humber R.A., Hyde K.D., Ironside J.E., Koljalg U., Kurtzman C.P., Larsson K.H., Lichtwardt R., Longcore J., Mia J., Miadlikowska J., Miller A., Moncalvo J.M., Mozley-Standridge S., Oberwinkler $F$., Parmasto E., Reeb V., Rogers J.D., Roux C., Ryvarden L., Sampaio J.A., Schübler A., Sugiyama J., Thorn R.G., Tibell L., Untereiner W.A., Walker C., Wang Z., Weir A., Weiss M., White M. M., Winka K., YAO Y.J., Zhang N., 2007 - A higher-level phylogenetic classification of the Fungi, Mycological Research, 111(5):509-547.

Hitchcock C. J., Chambers S. M. et Caimey J. W., 2010 - Genetic population structure of the ectomycorrhizal fungus Pisolithus microcarpus suggests high gene flow in south-eastern Australia. Mycorrhiza, 21(2): 131-7.

Hosaka K., S. T., Beever R. E., Castellano M. A. Colgan W., DominguezL. S. Nouhra E. R., Geml J., Admir J. Giachini A. J., Kenney S. R., Simpson N. B., Joseph W. Spatafora J. W. et Trappe J. M., 2006 - Molecular phylogenetics of the Gomphoid-Phalloid fungi with an establishment of the new sub-class Phallomycetideae and two new orders. Mycologia, 98: 949-959.

Houdou G., 2004 - Le grand livre des champignons. Ed. De Borée, $191 \quad p$ http://books.google.co.ma/books?id=U2AMoK EFHqkC\&dq=Tremella+mesenterica $\% 2 \mathrm{~B}+\mathrm{po}$ urriture+blanch

Ivancevic B. et Tatic B., 2004 - First record of Clathrus ruber from Serbia. Mycologia Balcancia, 1: 59 -60 .

Josserand M., 1983 - La description des champignons

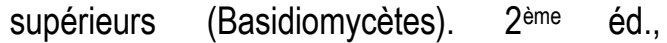
Lechevalier, Paris, 399 p.

Jülich W., 1981. - Higher taxa of Basidiomycetes. Bibl. Mycol., 85: 1-485.

Kirk M. P., Cannon P. F., Minter D. W. et Stalpers J. A., 2008 - Dictionary of the Fungi, 10ème édition. Edited by $\mathrm{P}$ M Kirk, International Mycological Institute, Egham, UK, P F Cannon, CABI, UK, J A Stalpers, CBS, The Netherlands September 2008 / Hardback / 784 Pages / 9780851998268 .

http://www.amazon.co.uk/Dictionary-Fungi10th-Kirk/dp/0851998267.

Kûhner R. et Romagnési H., 1984 - Flore analytique des champignons supérieurs (Agarics, Bolets, Chanterelles). Première édition, quatrième tirage, Masson, Paris, New York, Mexico, Sao Paulo, $556 \mathrm{p}$.

Lahayee E., 2006 - Rôle structurant des exopolysaccharides dans un biofilm bactérien. Thèse de Doctorat de l'Université de Bretagne Sud, France, $148 \mathrm{p}$.

Larouz B., 2007 - Biodiversité fongique du Maroc: Etude des champignons supérieurs du Moyen Atlas. Thèse de Doctorat. Faculté des Sciences, Université Ibn Tofaïl, Kénitra, Maroc, $322 p$.

Maire R. et Werner R. G., 1937 - Fungi Maroccani, catalogue raisonné des champignons connus jusqu'ici au Maroc. Mémoires de la Société 
des Sciences Naturelles, Empire Chérifien, Rabat - Paris - Londres, $143 \mathrm{p}$.

Malençon G. et Bertault R., 1956 - Champignons supérieurs de la région de Tanger (champignons récoltés de décembre 1955 à mars 1956: 2ème liste de Tanger). Comptes rendus mensuels des séances de la Société des Sciences Naturelles et Physiques du Maroc, T XXII, ${ }^{\circ} 5$. Publication subventionnée par l'Institut Scientifique Chérifien, Rabat, Maroc, pp 169-171.

Malençon G. et Bertault R., 1957 - Champignons de la région de Tanger (champignons récoltés de novembre 1956 à mai 1957: 3ème liste de Tanger). Comptes rendus mensuels des séances de la société des Sciences Naturelles et Physiques du Maroc, T. XXIII, $\mathrm{n}^{\circ} 8$. Publication subventionnée par l'Institut Scientifique Chérifien, Rabat, Maroc, pp 169_171.

Malençon G. et Bertault R., 1968 - Champignon de la région de Tanger et de Larache (9ème liste de Tanger et 1 ère liste de Larache). Bulletin de la Société des Sciences Naturelles et Physiques du Maroc, Rabat, T $47: 237$ - 250.

Malençon G. et Bertault R., 1969 - Champignons du Maroc II ; régions de Tanger et de Larache (10ème liste de Tanger et 2 ème liste de Larache). Bulletin de la Société des Sciences Naturelles et Physiques du Maroc, Rabat, T 49 : 71-72.

Malençon G. et Bertault R., 1969 - Champignons du plateau central et des subéraies de la Meseta. Deuxième liste, Comptes Rendus de la Société des Sciences Naturelles et Physique du Maroc, Bulletin de l'Institut Scientifique, Rabat, Maroc, pp 49-78.

Malençon G. et Bertault R., 1970 - Flore des champignons du Maroc, Tome I. Faculté des Sciences, Rabat, Maroc, $601 \mathrm{p}$.

Malençon G. et Bertault R., 1975 - Flore des champignons supérieurs du Maroc, T 2, Travaux de I'Institut Scientifique Chérifien et de la Faculté des Sciences de Rabat, série botanique et biologie végétale, $n^{\circ} 33$, Rabat, Maroc, $539 p$.

Marchand A., 1975. - Champignons du Nord et du Midi, Aphyllophorales, Hydnaceae, Gastéromycètes, Ascomycètes, T. 4, $162 \mathrm{p}$.

May T. W., Milne J., Sphingles S. et Jones R. H., 2003 - Fungi of Australia, volume 2B: Catalogue and Bibliography of Australian Fungi 2.
Basidiomycota p.p. \& Myxomycota, pp 133 247. http://www.publish.csiro.au/ or from: (c) Commonwealth of Australia, pp 133 - 247.

Pegler D. N. et Gomez L. D., 1994 - An unusual member of the cage fungus family. Mycologist, $8(2): 54-59$.

Petersoon C. H., 1818 - Traité sur les champignons comestibles contenant les espèces nuisibles. Imprimerie A. Egron, Paris, $305 \mathrm{p}$.

Pomperleau R., 1980 - Flore des champignons du Québec et des régions limitrophes. Ed. La Presse, $652 \mathrm{p}$.

Poumarat S., 2001 - Clé des Gastéromycètes épigés d'Europe: Lycoperdales, Nidulariales, Phallales, Sclérodematales, Tulostomatales. Monographies Mycologiques de la F.A.M.M., $\mathrm{n}^{\circ}$ 2. Editions Fédération des Associations Mycologiques Méditerranéennes, Nice, $96 \mathrm{p}$.

Romagnesi H., 1995 - Atlas des champignons d'Europe, ouvrage publié sous le patronage de la société Mycologique de France, éd. Bordas, Paris, $290 \mathrm{p}$.

Roux P., 2006 - Mille et un champignons. Editions Roux- Sainte Sigolène, France, $1223 p$.

Teixeira A. R., 1962 - The taxonomy of the Polyporaceae. Biol. Rev., 37: 51 - 81.

Tomšovsky M., ET Jankovsky L., 2007 - DNA sequence analysis of extraordinary fruiting specimens of Fuscoporia torulosa (Phellinus torulosus) on Pyrus spp. Czech Mycol., 59 (1): $91-99$.

Zeller S. M., 1949 - Notes on cetain Gasteromycetes including two new orders. Mycologia, 40: 639668. 